ROOK THEORY. I.

\title{
ROOK EQUIVALENCE OF FERRERS BOARDS
}

\author{
JAY R. GOLDMAN ${ }^{1}$, J. T. JOICHI AND DENNIS E. WHITE ${ }^{1}$
}

ABSTRACT. We introduce a new tool, the factorial polynomials, to study rook equivalence of Ferrers boards. We provide a set of invariants for rook equivalence as well as a very simple algorithm for deciding rook equivalence of Ferrers boards. We then count the number of Ferrers boards rook equivalent to a given Ferrers board.

Introduction. Let $\mathbf{N}$ denote the set of positive integers。 A board is a finite subset of $\mathbf{N} \times \mathbf{N}$. Intuitively, a board is an array of squares or cells arranged in rows and columns, i.e., a board is a subset of the set of squares of an $n \times n$ chessboard. We shall frequently utilize this intuitive terminology. We consider two boards to be the same board if one is a translate of the other, i.e., boards $B$ and $B^{\prime}$ are the same if there exist integers $a$ and $b$ such that $B^{\prime}=\{(i+a, j+b):(i, j) \in B\}$. Thus, only the geometric configuration or the relative positions of the cells is of significance.

For a board $B,|B|$ denotes the number of cells in $B$. Let $r_{k}^{B}$ be the number of ways of placing $k$ nontaking rooks (no two in the same row or column) on the board $B$, i.e., the number of $k$-subsets of the set $B$ such that no two elements of a $k$-subset have the same first component or the same second component. When no confusion can arise we suppress the $B$ and write $r_{k}$. The rook vector of a board $B$ is defined to be the vector $r(B)=$ $\left(r_{0}, r_{1}, r_{2}, \ldots\right)$ where $r_{0}=1$. Note that from some point on all the $r_{i}$ 's are zero, in particular $r_{i}=0$ for $i>|B|$. Two boards are called rook equivalent if they have the same rook vector.

$A$ board $B$ is a Ferrers board if there exists a nondecreasing finite sequence of positive integers $h_{1}, h_{2}, \ldots, h_{c}$ such that $B=\{(i, j): i \leq c$ and $\left.j \leq h_{i}\right\}$. Intuitively, a Ferrers board is a board made up of adjacent solid columns of cells with a common lower edge and such that the height of the columns from left to right forms a nondecreasing sequence. If the heights of the columns form a strictly increasing sequence, then we call the board an increasing Ferrers board. Examples of a Ferrers board and an increasing Ferrers board are given in Figures $1(\mathrm{a})$ and 1(b), respectively.

Received by the editors August 21, 1974.

AMS (MOS) subject classifications (1970). Primary 05A10, 05A15, 05 A19.

Key words and phrases. Rook numbers, rook equivalence, rook polynomials,

Ferrers board, permutations with restricted positions, binomial type, enumeration.

i Research supported in part by NSF Contract GP-43010. 


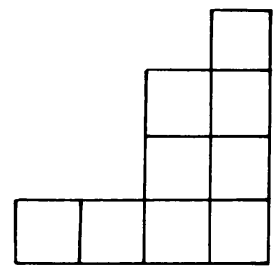

(a)

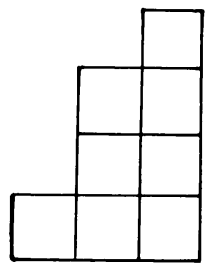

(b)

Figure 1

The theory of rook equivalence, as part of the theory of permutations with restricted positions, has its roots in the classical problems of derangements and Ménages as well as the works of MacMahon. For a systematic exposition of the field as of 1958 we refer to Riordan [8].

The first truly general theorem on rook polynomials was the classification of Ferrers boards by Foata and Schützenberger [3]. They proved that every Ferrers board is rook equivalent to a unique increasing Ferrers board. In some special cases they described the specific increasing board.

The principal tools for studying rook equivalence have been combinatorial arguments and the rook polynomial $\Sigma_{r_{k}} x^{k}$. In fact some of the motivation for the subject concerns the interesting classes of polynomials that are rook polynomials.

In this paper we introduce a new tool, the factorial polynomials, to study Ferrers boards. We provide a set of invariants for rook equivalence as well as a very simple algorithm for deciding rook equivalence of Ferrers boards. This allows us to prove the Foata-Schützenberger theorem using very simple constructions. We then count the number of Ferrers boards equivalent to a given Ferrers board.

Unless otherwise stated the term board shall always mean Ferrers board. The term Ferrers is added in the statement of theorems for emphasis.

I. The factorization theorem. For a Ferrers board $B$ with $c$ columns and column heights $h_{1} \leq h_{2} \leq \cdots \leq h_{c}$, we define the height vector $h(B)$ to be $h(B)=\left(h_{1}, h_{2}, \ldots, h_{c}\right)$ and $n \geq c$, the $n$-height vector $h_{n}(B)$ to be $h_{n}(B)$ $=\left(h_{1}^{(n)}, h_{2}^{(n)}, \ldots, h_{n}^{(n)}\right)$ where $h_{i}^{(n)}=0$ for $i=1,2, \ldots, n-c$ and $h_{i}^{(n)}=$ $h_{i-(n-c)}$ for $i=n-c+1, \ldots, n$. We note that $h_{c}(B)=h(B)$ and that $h_{n}(B)$ corresponds to thinking of a set of $n-c$ "empty" columns to the left of $B$. The crucial quantities in the study of a Ferrers board $B$ are the associated $n$-structure vectors $s_{n}(B)$ which are defined to be $s_{n}(B)=\left(s_{1}^{(n)}, s_{2}^{(n)}, \ldots\right.$, $\left.s_{n}^{(n)}\right)$ where $s_{i}^{(n)}=h_{i}^{(n)}-(i-1)$. In the example of Figure 1(a) we have $h(B)=(1,1,3,4), h_{6}(B)=(0,0,1,1,3,4)$ and $s_{6}(B)=(0,-1,-1,-2,-1$, $-1)$.

The following proposition is immediate. 
1. Proposition. The n-structure vector of a Ferrers board satisfies the conditions

(i) $s_{1}^{(n)} \geq 0$

(ii) $s_{i}^{(n)} \geq s_{i-1}^{(n)}-1$ for $i=2,3, \ldots, n$, and

(iii) $s_{i}^{(n)}=s_{i-1}^{(n)}-1$ if and only if $h_{i}^{(n)}=h_{i-1}^{(n)}$. Conversely, any sequence of $n$ integers satisfying (i) and (ii) is the n-structure vector of a uniquely determined Ferrers board.

To study the structure of the rook vectors, we associate with each board $B$ the sequence $\left\langle p_{n}(x, B)\right\rangle_{n \geq c}$ of $n$-factorial polynomials defined by $p_{n}(x, B)=\sum_{k=0}^{n} r_{k} \cdot(x)_{n-k}$ where $(x)_{i}=x(x-1)(x-2) \ldots(x-i+1)$ is the falling factorial.

The key result of this paper is the

2. Factorization theorem. If $B$ is a Ferrers board with $c$ columns and has, for $n \geq c$, n-structure vector $s_{n}(B)=\left(s_{1}^{(n)}, \ldots, s_{n}^{(n)}\right)$ and $n$-factorial polynomial $p_{n}(x, B)$, then

$$
p_{n}(x, B)=\prod_{i=1}^{n}\left(x+s_{i}^{(n)}\right) .
$$

Proofo Let $B$ have height vector $h(B)=\left(h_{1}, \ldots, h_{c}\right)$ and $n$-height vector $h_{n}(B)=\left(h_{1}^{(n)}, \ldots, h_{n}^{(n)}\right)$. For $x$ a positive integer, let $B_{x, n}$ be the Ferrers board with height vector

$$
h\left(B_{x, n}\right)=\left(h_{1}^{\prime}, h_{2}^{\prime}, \ldots, h_{n}^{\prime}\right)=\left(x^{\prime}, x, \ldots, x, x+b_{1}, x+h_{2}, \ldots, x+h_{c}\right) .
$$

Thus, $B_{x, n}$ is obtained from $B$ by attaching an $x \times n$ rectangle to the lower edge of $B$ aligning the right-hand columns (Figure 2). We now count the number of ways of placing $n$ nontaking rooks on $B_{x, n}$ in ${ }^{-}$two different ways.

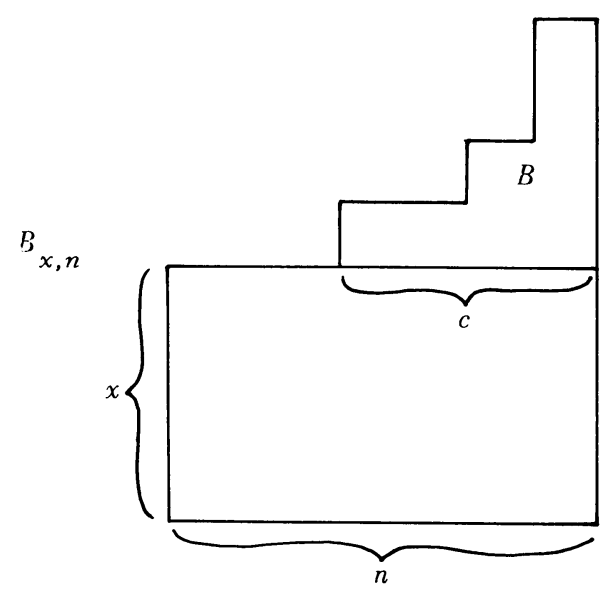

Figure 2 
First we break the count up into cases by the number of rooks placed on $B$. If $k$ rooks are placed on $B$, this rules out $k$ of the columns of the $x \times n$ rectangle and the remaining $n-k$ rooks can be placed on the remaining $x \times(n-k)$ rectangle in $x(x-1)(x-2) \ldots(x-n+k+1)=(x)_{n-k}$ ways. Hence, $r_{k} \cdot(x)_{n-k}$ counts the number of ways of placing $n$ rooks on $B_{x, n}$ such that $k$ of them are placed on $B$ and $p_{n}(x, B)=\Sigma_{k=0}^{n} r_{k} \cdot(x)_{n-k}$ is the number of ways of placing $n$ nontaking rooks on $B_{x, n}$.

We can also count the number of rook placings on $B_{x, n}$ by placing them in one column at a time from left to right. A rook can be placed in the first column in $x+h_{1}^{(n)}=x+s_{1}^{(n)}$ ways. This rules out one row and a rook can be placed in the second column in $x+h_{2}^{(n)}-1=x+s_{2}^{(n)}$ ways. Continuing in this manner we see that $n$ nontaking rooks can be placed on $B_{x, n}$ in $\Pi_{i=1}^{n}\left(x+s_{i}^{(n)}\right)$ ways.

Equating the results of our two counts, we have the desired result for all positive integral values of $x$, and hence, we have a polynomial identity.

The following two corollaries are immediate.

3. Corollary. Two Ferrers boards are rook equivalent if and only if, for some $n$, their n-factorial polynomials are equal.

Recall that a multiset is a "set" that can have repeated identical elements. More formally, a multiset is a set together with a positive integer valued function defined on the set (which can be thought of as giving the multiplicities of the elements). Since an $n$-structure vector $s_{n}(B)$ can have repeated components, the collection of its components forms a multiset. We denote this multiset by $S_{n}(B)$.

4. Corollary. Two Ferrers boards $B$ and $B^{\prime}$ are rook equivalent if and only if, for some $n, S_{n}(B)=S_{n}\left(B^{\prime}\right)$.

II. Rook equivalence and enumeration of Ferrers boards. We now characterize the rook equivalence classes of Ferrers boards by picking a unique representative from. each class. Also we count the number of Ferrers boards rook equivalent to a given Ferrers board.

5. Theorem (Foata-Schützenberger [3]). Every Ferrers board is rook equivalent to a unique increasing Ferrers board.

Proof. First we examine the $n$-structure vector $s_{n}(B)$ of an increasing Ferrers board $B$ with $c$ columns and column heights $h_{1}<h_{2}<\cdots<h_{c}$. Since $h_{n}(B)=\left(0,0, \ldots, 0, h_{1}, \ldots, h_{c}\right)$ where we have $n-c$ initial zeroes, we will have 


$$
s_{n}(B)=\left(0,-1,-2, \ldots,-(n-c-1), u_{1}, u_{2}, \ldots, u_{c}\right)
$$

where $-(n-c-1) \leq u_{1} \leq u_{2} \leq \cdots \leq u_{c}$. Furthermore, by Proposition 1, every such vector is the $n$-structure vector of a uniquely determined increasing Ferrers board.

Now let $B$ be an arbitrary Ferrers board. We shall construct an increasing Ferrers board $B^{\prime}$ rook equivalent to $B$. Since $r_{1}^{B}=|B|$, all boards equivalent to $B$ have the same number of cells as $B$. Let $N \stackrel{\bullet}{=}|B|+1$ and consider the $N$-structure vector $s_{N}(B)$. We have $s_{1}^{(N)}=0$. Let $-t$ be the smallest component of $s_{N}^{(B)}$. Then, by Proposition 1 , the elements $-1,-2, \ldots$, $-(t-1)$ must also appear among the components of $s_{N}(B)$. Define the vector $q$ by

$$
q=\left(0,-1,-2, \ldots,-(t-1),-t, q_{1}, q_{2}, \ldots, q_{N-t-1}\right)
$$

where the $q_{i}$ 's are the elements of $S_{N}(B)$ with $\{0,-1, \ldots,-t\}$ deleted and listed in nondecreasing order. By the discussion of the preceding paragraph, $q$ is the $N$-structure vector of an increasing Ferrers board $B^{\prime}$. Since $S_{N}(B)$ $=S_{N}\left(B^{\prime}\right), B$ and $B^{\prime}$ are rook equivalent by Corollary 4. Furthermore, since - $t$ is the minimum element of $S_{N}(B)$, it follows that $B^{\prime}$ is the only increasing Ferrers board rook equivalent to $B$.

The constructive nature of the preceding proof allows us to go much further and actually count the set of Ferrers boards equivalent to a given board $B$. In order to do this, we assume familiarity with the basic concepts of permutations of a multiset, a theory developed by Foata [2]. For a very clear exposition of this development, see $K_{\text {nuth [6]. }}$

The vector $s_{N}(B)$ is a permutation of the multiset $S_{N}(B)$. By Corollary 4 , a board $B^{\prime}$ is equivalent to $B$ if and only if $s_{N}\left(B^{\prime}\right)$ is also a permutation of $S_{N}(B)$. Hence, counting all boards equivalent to $B$ is the same as counting all permutations of $S_{N}(B)$ subject to the conditions (i) and (ii) of Proposition 1. Since $N=|B|+1$, we may replace (i) by the condition that the first element of the permutation is always zero. From $N>|B|$, it also follows by an elementary count, that $s_{i}^{(N)} \leq 0$ for all $i$.

Now using the Foata correspondence [6, p. 27, Theorem B] between multiset permutations in one and two line notations, our problem is equivalent to counting the number of permutations of $S_{N}(B)$ in two line notation such that the left-most bottom element is zero and the pair ${ }_{m}^{k}$ cannot occur for $m<k-1$. This is an easy count using binomial coefficients and leads to the following

6. Theorem. If $B$ is a Ferrers board and $-t$ is the smallest component of $s_{|B|+1}(B)$, then the number of Ferrers boards rook equivalent to $B$ is equal to 


$$
\left(\begin{array}{c}
j_{0}+j_{1}-1 \\
j_{1}
\end{array}\right)\left(\begin{array}{c}
j_{1}+j_{2}-1 \\
j_{2}
\end{array}\right) \cdots\left(\begin{array}{c}
j_{t-1}+j_{t}-1 \\
j_{t}
\end{array}\right)
$$

where $j_{i}$ is the multiplicity of $-i$ in $S_{|B|+1}(B)$.

Since the vector $S_{|B|+1}(B)$ is obtained by adjoining many zeroes to the height vector, this often leads to the preceding expression having a large number of factors. To make computation more convenient we look at the increasing board in an equivalence class and consider $S_{C}(B)$ where $c$ is the number of columns of $B$. This leads to the following

7. Corollary. If $B$ is an increasing Ferrers board with $c$ columns, then the number of Ferrers boards rook equivalent to $B$ is

$$
\left(\begin{array}{c}
a_{1}+a_{2}+1 \\
a_{2}
\end{array}\right)\left(\begin{array}{c}
a_{2}+a_{3}+1 \\
a_{3}
\end{array}\right) \cdots\left(\begin{array}{c}
a_{u-1}+a_{u}+1 \\
a_{u}
\end{array}\right)
$$

where $a_{i}$ is the multiplicity of $i$ in $S_{c}(B)$ and $u=s_{c}^{(c)}$.

We can in fact do more than count the equivalence class. Given a board $B$, by using the usual backtrack algorithm [5], we can list all permutations of $S_{n}(B)$ satisfying the conditions of Proposition 1 . This gives us an effective procedure for listing all boards equivalent to $B$.

III. Some special boards. We call a board $B$ an $m$-jump board if $h(B)$ $=(m, 2 m, 3 m, \ldots)$.

8. Proposition. Let $B$ be an $(m+1)$-jump board with $n-1$ columns. Then the $n$-factorial polynomial is given by $p_{n}(x, B)=x^{(n, m)}$ where $x^{(n, m)}$ $=x(x+m)(x+2 m) \ldots(x+(n-1) m)$ is the m-step rising factorial.

Proof. We have $h_{n}(B)=(0, m+1,2(m+1), \ldots,(n-1)(m+1))$ and thus $s_{n}(B)=(0, m, 2 m, \ldots,(n-1) m)$ and the result follows from the factorization theorem.

This proposition gives us an interesting combinatorial interpretation of the $m$-step rising factorial as a factorial polynomial. It is well known [7] that $x^{(n, m)}$ is of binomial type, i.e.,

$$
(x+y)^{(n, m)}=\sum_{k=0}^{n}\left(\begin{array}{l}
n \\
k
\end{array}\right) x^{(k, m)} y^{(n-k, m)} .
$$

This can be proved combinatorially using our interpretation of $x^{(n, m)}$. We also mention that $x^{(n, m)}$ can be proved to be of binomial type using a combinatorial argument in the spirit of reluctant functions [7]. 
For the 1-jump board $B$ with $n-1$ columns we have $p_{n}(x, B)=x^{n}=$ $\Sigma_{k^{r} k} \cdot(x)_{n-k}$. The well-known identity $x^{n}=\Sigma_{k} S(n, k) \cdot(x)_{k}$, where $S(n, k)$ are Stirling numbers of the second kind (see [1], [9]), yields $r_{k}=S(n, n-k)$. We observe, by Corollary 7 , that this board is not rook equivalent to any other Ferrers board.

For the 2-jump board $B$ with $n-1$ columns we have $p_{n}(x, B)=x^{(n, 1)}$ $\left(=x^{(n)}\right.$, the rising factorial). Since $s_{n}(B)=(0,1,2, \ldots, n-1)$, the new vector $(n-1, n-2, \ldots, 1,0)$ determines a rook equivalent board, namely, the $(n-1) \times n$ rectangle. By Corollary 7 , the number of boards rook equivalent to $B$ is $2 \cdot 3^{n-2}$.

For $m>2$, by Corollary 7 , the number of boards rook equivalent to the $m$-jump board with $n-1$ columns is $2^{n-1}$.

IV. Comments. We can, in general, define the factorial polynomial for an arbitrary board $B$ and the combinatorial interpretation in terms of $B_{x, n}$ carries over. However, there does not seem to be a factorization in cases other than the Ferrers boards.

Riordan's "associated" rook polynomial [8] $\Sigma_{k} r_{k} x^{n-k}$ of a board $B$ can also be interpreted in terms of $B_{x, n}$ by a modified definition of rook placing.

The Foata-Schützenberger correspondence [4, p. 38] between the 1-jump board and partitions of a set can be generalized to correspondences between general Ferrers boards and partitions of a set with special restrictions.

A formal development of "factorial hit polynomials" analogous to the classical formulas [8] can be given where the operator $\Delta(\Delta f(x)=f(x+1)$ $f(x))$ replaces the differentiation operator $D$.

The theory of rook numbers of $m$-jump boards can be developed from both analytic and combinatorial viewpoints and has an intimate connection with the theory of binomial enumeration [7]. This will be published elsewhere.

\section{BIBLIOGRAPHY}

1. L. Comtet, Analyse combinatoire. Tomes I, II, Collection SUP: Le Mathématicien, 4, 5, Presses Universitaires de France, Paris, 1970. MR 41 \#6697.

2. D. C. Foata, Etude algébrique de certains problèmes d'analyse combinatoire et du calcul des probabilités, Publ. Inst. Statist. Univ. Paris 14 (1965), 81-241. MR $36 \# 3392$.

3. D. C. Foata and M. P. Schützenberger, On the rook polynomials of Ferrers relations, Colloq. Math. Soc. Janos Bolyai, 4, Combinatorial Theory and its Applications, vol. 2 (P. Erdös et al., eds.), North-Holland, Amsterdam, 1970.

4. - Théorie géométrique des polynômes eulériens, Lecture Notes in Math., vol. 138, Springer-Verlag, Berlin and New York, 1970. MR 42 \#7523.

5. S. W. Golomb and L. D. Baumert, Backtrack programming, J. Assoc. Comput. Mach. 12 (1965), 516-524. MR 33 \#3783.

6. D. E. Knuth, The art of computer programming. Vol. 3: Sorting and searching, AddisonoWesley, Reading, Mass., 1968. 
7. R. C. Mullin and G.-C. Rota, On the foundations of combinatorial theory. III. Theory of binomial enumeration, Graph Theory and its Applications (Proc. Advanced Sem., Math. Res. Center, Univ. of Wisconsin, Madison, Wis., 1969), Academic Press, New York, 1970, pp. 167-213. MR 43 \#65.

8. J. Riordan, An introduction to combinatorial analysis, Wiley, New York; Chapman \& Hall, London, 1958. MR 20 \#3077.

9. G.-C. Rota, The number of partitions of a set, Amer. Math. Monthly 71 (1964), 498-504. MR $28 \# 5009$.

SCHOOL OF MATHEMATICS, UNIVERSITY OF MINNESOT A, MINNE APOLIS, MINNESOTA 55455 